\title{
COMPARISON THEOREMS FOR SPECIAL CLASSES OF NONSELFADJOINT ELLIPTIC EQUATIONS
}

\author{
KURT KREITH ${ }^{1}$
}

\begin{abstract}
Known comparisons for nonselfadjoint elliptic equations are strengthened in cases where the coefficients of these equations satisfy special conditions. These improved comparison theorems are also considered in the context of a related eigenvalue problem.
\end{abstract}

1. Introduction. In [1] a class of Sturmian comparison theorems is established for nonselfadjoint elliptic equations of the form

$$
\begin{aligned}
l u & \equiv-\nabla \cdot a(x)(\nabla u)^{T}+2 b(x)(\nabla u)^{T}+c(x) u=0, \\
L v & \equiv-\nabla \cdot A(x)(\nabla v)^{T}+2 B(x)(\nabla v)^{T}+C(x) v=0,
\end{aligned}
$$

whose coefficients are real and defined in a domain $D \subset E^{n}$. These theorems, to be restated below, assume no special relationship among the coefficients of (1) and (2) nor any special condition other than smoothness and ellipticity. The present paper is concerned with some stronger theorems which can be established when certain special conditions or relationships are assumed.

The formulation and proofs of such theorems are simplified by the matrix notation $a=\left(a_{i j}\right)$ and $A=\left(A_{i j}\right)$ for $i, j=1, \cdots, n$ and the vector notation $\boldsymbol{b}=\left(b_{1}, \cdots, b_{n}\right)$ and $\boldsymbol{B}=\left(B_{1}, \cdots, B_{n}\right)$. Inequalities for matrices are to be interpreted in terms of positive definiteness. Thus in (1) it is assumed that $a(x), b(x)$, and $c(x)$ are of class $C^{2}, C^{1}$ and $C$, respectively, and that $a=a^{*}>0$; analogous assumptions are made with regard to (2).

Given a nodal domain $D$ for a solution $u(x)$ of $(1)$, conditions are sought which assure that every solution $v(x)$ of (2) must have a zero in $\bar{D}$. Such conditions involve functions $g(x)$ and $G(x)$ defined by

$$
g=b a^{-1} b^{T}, \quad G=B A^{-1} B^{T},
$$

and require that $L$ be appropriately majorised by $l$. Specifically, the

Received by the editors November 27, 1972 and, in revised form, March 30, 1973. AMS (MOS) subject classifications (1970). Primary 35B05.

Key words and phrases. Sturmian comparison theorem, nodal domain, conservative vector field, solenoidal vector field, eigenvalue.

${ }^{1}$ Research supported by a grant of the Science Research Council. 
techniques of [1] show that if

$$
\begin{aligned}
\iint_{D}\left[\nabla u(a-A)(\nabla u)^{T}+2 u(b-B\right. & +p)(\nabla u)^{T} \\
& \left.+\left(c-C-G+\nabla \cdot p^{T}\right) u^{2}\right] d x \geqq 0
\end{aligned}
$$

for some $C^{1}$ vector function $p(x)$, then the conditions of a Sturmian comparison theorem are satisfied. Choosing $p(x) \equiv 0$, (4) follows from

(5) $a>A>0$,

$$
c-C-G-(b-B)(a-A)^{-1}(b-B)^{T} \geqq 0,
$$

conditions (5) having previously been established by Dunninger [2] and the author [3]. Choosing $p(x)=B(x)-b(x)$, (4) follows from

$$
a \geqq A>0, \quad c-C-G+\nabla \cdot(B-b) \geqq 0,
$$

conditions (6) having previously been established by Swanson [4] and Allegretto [5].

An example of a stronger result which can be established in special cases is contained in [6]. Here it is shown that if $a(x) \equiv A(x)$ and $b(x) \equiv$ $\boldsymbol{B}(x)$ in $D$, then the condition

$$
c-C \geqq 0
$$

assures the validity of a Sturmian comparison theorem for (1) and (2). In other words, under the above special assumption, the nonnegative term $G(x)$ may be dropped in (6).

A number of the theorems in this vein will be established below. The results will also be considered in the context of a related eigenvalue problem studied by Allegretto [7].

2. Conservative vector fields. Our basic observation is that the substitution $u(x)=P(x) U(x)$ transforms (1) into

$$
\begin{aligned}
l u \equiv & -P \nabla \cdot a(\nabla U)^{T}+2(P b-\nabla P a)(\nabla U)^{T} \\
& +\left(c P-\nabla \cdot a(\nabla P)^{T}+2 b \cdot(\nabla P)^{T}\right) U=0,
\end{aligned}
$$

while $v(x)=R(x) V(x)$ transforms (2) into

$$
\begin{aligned}
L v \equiv & -R \nabla \cdot A(\nabla V)^{T}+2(R B-\nabla R A)(\nabla V)^{T} \\
& +\left(C R-\nabla \cdot A(\nabla R)^{T}+2 B \cdot(\nabla R)^{T}\right) V=0 .
\end{aligned}
$$

These transformations are especially important in case $b(x) a^{-1}(x)$ and $\boldsymbol{B}(x) A^{-1}(x)$ are conservative vector fields-i.e. if there exist $C^{2}$ functions $f(x)$ and $F(x)$ such that

$$
b a^{-1}=\nabla f, \quad B A^{-1}=\nabla F .
$$


THEOREM 1. If $D$ is a nodal domain for a solution $u(x)$ of $(1)$, and if in $D$

(i) $a \geqq A>0$,

(ii) $\boldsymbol{b} \boldsymbol{a}^{-1}=\nabla f, B A^{-1}=\nabla F$,

(iii) $c-C+g-G-\nabla \cdot(b-B) \geqq 0$,

then every solution $v(x)$ of $(2)$ has a zero in $D$.

Proof. Choosing $P(x)=e^{f(x)}$ and $R(x)=e^{F(x)},\left(1^{\prime}\right)$ and $\left(2^{\prime}\right)$ become

$$
l u \equiv P\left[-\nabla \cdot a(\nabla U)^{T}+(c+g-\nabla \cdot b) U\right]=0
$$

and

$$
L v \equiv R\left[-\nabla \cdot A(\nabla V)^{T}+(C+G-\nabla \cdot B) V\right]=0,
$$

respectively. Thus $U(x)$ and $V(x)$ satisfy selfadjoint elliptic equations obtained by setting the expressions inside the brackets equal to zero. Applying well-known comparison theorems for selfadjoint elliptic equations (see for example [8]) and making use of the fact that $U(x)$ and $V(x)$ have the same oscillation properties as $u(x)$ and $v(x)$ respectively, the theorem follows.

Thus if the coefficients of (1) and (2) satisfy (8), then the nonnegative expression $g(x)$ may be added to the left side of the second inequality in (6), thereby strengthening the comparison theorems of [1] and [4].

In case $b a^{-1}$ and $B A^{-1}$ are not conservative, they may still have conservative components allowing a decomposition of the form

$$
b a^{-1}=\nabla f+s, \quad B A^{-1}=\nabla F+S .
$$

In this case the substitutions $u=e^{f} U$ and $v=e^{F} V$ yield

$$
\begin{aligned}
e^{-f} l u \equiv & -\nabla \cdot a(\nabla U)^{T}+2 s a(\nabla U)^{T} \\
& +\left(c-\nabla \cdot a(\nabla f)^{T}+g-s a s^{T}\right) U=0
\end{aligned}
$$

and

$$
\begin{aligned}
e^{-F} L v \equiv & -\nabla \cdot A(\nabla V)^{T}+2 S A(\nabla V)^{T} \\
& +\left(C-\nabla \cdot A(\nabla F)^{T}+G-S A S^{T}\right) V=0,
\end{aligned}
$$

to which previously cited comparison theorems can be applied. One case of special interest is that where $a(x) \equiv A(x)$ and $s(x) \equiv S(x)$ so that $b a^{-1}$ $B A^{-1}$ is conservative. In this case (7) yields the following.

THEOREM 2. If $D$ is a nodal domain for a solution $u(x)$ of $(1)$, and if in $D$

(i) $a=A$,

(ii) $B A^{-1}-b a^{-1}=\nabla h$,

(iii) $c-C+g-G+\nabla \cdot(B-b) \geqq 0$,

then every solution $v(x)$ of $(2)$ has a zero in $D$. 
In case $A=I$ and the vector fields $s$ and $S$ are solenoidal, then

$$
\nabla \cdot a(\nabla f)^{T}=\nabla \cdot b \text { and } \nabla \cdot A(\nabla F)^{T}=\nabla \cdot B .
$$

An application of (6) to equations (10) and (11) then yields the following.

THEOREM 3. If $D$ is a nodal domain for a solution $u(x)$ of $(1)$ and if in $D$

(i) $a=A=I$,

(ii) $b=\nabla f+s$, where $\nabla \cdot s=0 ; B=\nabla F+S$, where $\nabla \cdot S=0$,

(iii) $c-C+g-G+\nabla \cdot(B-b)-s s^{T} \geqq 0$,

then every solution $v(x)$ of $(2)$ has a zero in $D$.

In case $\nabla f=\nabla F=0, g=s s^{T}$ and condition (iii) of Theorem 3 reduces to

(iii) $c-C-S S^{T} \geqq 0$.

3. Bounds for eigenvalues. Comparison theorems for (1) and (2) are intimately related to eigenvalue problems of the form

$$
l u=\lambda u, \quad \text { in } D, \quad u=0, \text { on } \partial D,
$$

and

$$
L v=\Lambda v, \quad \text { in } D, \quad v=0 \text {, on } \partial D .
$$

This relation follows from the fact that (11) has a distinguished real simple eigenvalue $\lambda_{0}$ which is smaller than any other real eigenvalue. The eigenfunction $u_{0}(x)$ corresponding to $\lambda_{0}$ may be taken positive in $D$ while all other eigenfunctions change sign in $D ;(12)$ has a distinguished eigenvalue $\Lambda_{0}$ with corresponding properties. The conclusion of the above comparison theorems (to the effect that solutions of (2) have a zero in $\bar{D}$ ) is equivalent to the inequality $\Lambda_{0} \leqq \lambda_{0}$ (see [7]).

Allegretto [7] has studied the relationship between the distinguished eigenvalues of (11) and (12) where $L$ is given by (2) and the operator of (11) is of the form $l_{q}=\frac{1}{2}\left(L+L^{*}\right)+q$ so that (11) becomes

$$
\begin{aligned}
l_{q} u & \equiv-\nabla \cdot A(\nabla u)^{T}+(C-\nabla \cdot B+q) u=\lambda u, \text { in } D, \\
u & =0, \text { on } \partial D .
\end{aligned}
$$

Letting $\lambda_{0}(q)$ denote the smallest eigenvalue of the operator defined by $\left(11^{\prime}\right)$ it follows readily from the variational characterization of $\lambda_{0}$ that $\lambda_{0}(0) \leqq \Lambda_{0}$. On the other hand, it follows from (6) that $\Lambda_{0} \leqq \lambda_{0}(G)$ where $G=B A^{-1} B^{T}$. Thus, as also shown in [7], we always have

$$
\lambda_{0}(0) \leqq \Lambda_{0} \leqq \lambda_{0}(G)
$$

The transformation of (2) into (2") yields the following result first established by Allegretto [5, Corollary 6].

THEOREM 4. If $B A^{-1}=\nabla F$ then $\Lambda_{0}=\lambda_{0}(G)$. 
There remains the question of the effect of a solenoidal component $S$ on estimates for $\Lambda_{0}$. The following example shows that the addition of a solenoidal component to $B$ may not affect $\Lambda_{0}$. The equation

$$
-\Delta v+\frac{2 x}{x^{2}+y^{2}} v_{x}+\frac{2 y}{x^{2}+y^{2}} v_{y}-4\left(x^{2}+y^{2}\right) v=0
$$

has solution $v(x, y)=\cos \left(x^{2}+y^{2}\right)$. Choosing

$$
D=\left\{(x, y) \mid \pi / 2<x^{2}+y^{2}<3 \pi / 2\right\}
$$

it follows that (12) with

$$
\begin{aligned}
2 B & =\left(2 x /\left(x^{2}+y^{2}\right), 2 y /\left(x^{2}+y^{2}\right)\right)=\nabla \log \left(x^{2}+y^{2}\right), \\
C & =-4\left(x^{2}+y^{2}\right)
\end{aligned}
$$

satisfies $\Lambda_{0}=0$. Here $G=B B^{T}=1 /\left(x^{2}+y^{2}\right)$, so that by Theorem 4

$$
\lambda_{0}\left(\frac{1}{\left(x^{2}+y^{2}\right)}\right)=\Lambda_{0}=0 \text {. }
$$

However for any constant $k$ the equation

$$
-\Delta v+\frac{2 x+k y}{x^{2}+y^{2}} v_{x}+\frac{2 y-k x}{x^{2}+y^{2}} v_{y}-4\left(x^{2}+y^{2}\right) v=0
$$

also has the solution $v(x, y)=\cos \left(x^{2}+y^{2}\right)$. Thus (12) with

$$
\begin{aligned}
& \boldsymbol{B}=\nabla\left[\frac{1}{2} \log \left(x^{2}+y^{2}\right)\right]-\frac{1}{2} k(-y, x), \\
& C=-4\left(x^{2}+y^{2}\right),
\end{aligned}
$$

also satisfies $\Lambda_{0}=0$. However now $G=B B^{T}=\left(\frac{1}{2} k^{2}+1\right) /\left(x^{2}+y^{2}\right)$ so that if $k \neq 0$, then $\lambda_{0}(G)>\Lambda_{0}$.

It would be of interest to obtain a general theorem which sharpens the estimate (13) in terms of the conservative and nonconservative components of $B A^{-1}$.

ADDED IN PROOF. A simplified and more general proof of (7) has recently been given by C. Y. Chan (Bull. London Math. Soc. (to appear)). Condition (4) also follows from an identity of C. A. Swanson (Proc. Amer. Math. Soc. 37 (1973), 537-540).

\section{REFERENCES}

1. K. Kreith, A class of comparison theorems for nonselfadjoint elliptic equations, Proc. Amer. Math. Soc. 29 (1971), 547-552. MR 43 \#5140.

2. D. Dunninger, A Picone identity for nonselfadjoint elliptic operators, Atti Accad. Naz. Lincei Mem. Cl. Sci. Fis. Mat. Natur. Sez. I 48 (1970), 135-139. 
3. K. Kreith, A comparison theorem for general elliptic equations with mixed boundary conditions, J. Differential Equations 8 (1970), 537-541. MR 42 \#646.

4. C. A. Swanson, A comparison theorem for elliptic equations, Proc. Amer. Math. Soc. 17 (1966), 611-616. MR 34 \#1663.

5. W. Allegretto, A comparison theorem for nonlinear operators, Ann. Scuola Norm. Sup. Pisa 25 (1971), 41-46.

6. K. Kreith, Applications of a comparison theorem for elliptic equations, Illinois J. Math. 15 (1971), 47-51. MR 42 \#8056.

7. W. Allegretto, Eigenvalue comparison and oscillation criteria for elliptic operators, J. London Math. Soc. (2) 3 (1971), 571-575. MR 44 \#1911.

8. K. Kreith, A strong comparison theorem for selfadjoint elliptic equations, Proc. Amer. Math. Soc. 19 (1968), 989-990. MR 37 \#3178.

Department of Mathematics, Chelsea College, manresa Road, London, ENGLAND

Current address: Department of Mathematics, University of California, Davis, California 95616 